\title{
The size-dependent elastic properties of nanofilms with surface effects
}

\author{
Jian-Gang Guo and Ya-Pu Zhao ${ }^{\text {a) }}$ \\ State Key Laboratory of Nonlinear Mechanics (LNM), Institute of Mechanics, Chinese Academy of Sciences, \\ Beijing 100080, People's Republic of China
}

(Received 2 February 2005; accepted 24 August 2005; published online 5 October 2005)

\begin{abstract}
Size-dependent elastic constants are investigated theoretically with reference to a nanoscale single-crystal thin film. A three-dimensional (3D) model is presented with the relaxation on the surface of the nanofilm taken into consideration. The constitutive relation of the 3D model is derived by using the energy approach, and analytical expressions for the four nonzero elastic constants of the nanofilm are obtained. The size effects of the four elastic constants are then discussed, and the dependence of these elastic constants on the surface relaxation and the ambiguity in the definition of the thickness of the nanofilm are also analyzed. In addition, the elastic moduli of the nanofilm in two kinds of plane problem are obtained and discussed in the case of a special boundary condition. (C) 2005 American Institute of Physics. [DOI: 10.1063/1.2071453]
\end{abstract}

\section{INTRODUCTION}

With the rapid development of nanoelectromechanicalsystem (NEMS) technologies, ${ }^{1}$ ultrathin films (with the thickness of the film in the nanoscale) have been extensively used as structural as well as electrical components of NEMS devices, especially ultrasensitive sensors for ultrafine resolution applications. For long-term reliability of various NEMS devices, it is necessary to understand the mechanical properties of ultrathin films including hardness, Young's modulus, Poisson's ratio, etc. Like many other properties, the mechanical properties of ultrathin films deviate from macroscopic ones as the characteristic dimensions become very small, which means that we cannot apply the macroscopic properties anymore to the nanoscale systems such as nanofilm, NEMS, etc. Thus, the size effects of the mechanical properties at the nanoscale are important, and an analytical model is desirable.

A great deal of research has been done on the elastic characteristics of micro- and nanoscale materials including experiments, theoretical analysis, and atomistic or moleculardynamics simulation. Many experimental methods have been developed to evaluate Young's modulus of thin films, including nanoindenter techniques, ${ }^{2-5}$ atomic force microscopy (AFM) techniques, ${ }^{6-10}$ x-ray diffraction with in situ tensile testing, ${ }^{11,12}$ the measurement of resonant frequencies of ultrathin cantilever resonators, ${ }^{13,14}$ laser-ultrasonic surface acoustic wave (SAW) spectrometry, ${ }^{15}$ as well as so-called free overhang method. ${ }^{16}$ Besides the classical continuum theory, ${ }^{17-25}$ the $a b$ initio calculation was used to study the elastic constants of nanostructures. ${ }^{26}$ In addition, atomistic or molecular simulation ${ }^{27-31}$ was also extensively applied to analyze the mechanical properties of nanocrystals. It has been found that the hardness is size dependent on the microscale, but Young's modulus is size independent when the characteristic scale is greater than $500 \mathrm{~nm}$ (Refs. 5, 11, and 14) or $200 \mathrm{~nm}^{6}$ What happens to Young's modulus when the characteristic scale is smaller than $200 \mathrm{~nm}$ or even far

\footnotetext{
${ }^{\text {a) }}$ Author to whom correspondence should be addressed; FAX: 86106256 1284; electronic mail: yzhao@lnm.imech.ac.cn
}

smaller? It has been proved in many experiments that the elastic constants are size dependent at the nanoscale. Some experiments show that Young's modulus and Poisson's ratios increase with the decrease of the characteristic dimension of materials, $4,7,8,11$ while others show that the reverse is true. . $^{2,10,12,13,15,16}$ Theoretical investigations also reach the two opposite conclusions. Krivtsov and Morozov ${ }^{18}$ studied theoretically the size-dependent elastic moduli of a nanocrystal with reference to a two-dimensional (2D) strip of a single crystal with hexagonal-closely-packed structure, and it was concluded that Poisson's ratio decreases and Young's modulus increases with the decreasing thickness of a single-crystal strip. For a very thin crystal film (of two atomic layers thick), these elastic moduli can differ from their macroscopic values by a factor of 2. Liang et al. ${ }^{19}$ established a model for the size-dependent elastic moduli of $\mathrm{Cu}$ and $\mathrm{Au}$ thin films and predicted the enhancement of the elastic moduli of $\mathrm{Cu}$ and $\mathrm{Au}$ thin films. $\mathrm{Yang}^{25}$ and Streitz et $a .^{27}$ analyzed, respectively, the effects of surface energy and surface stress on the elastic properties of nanomaterials and reached similar conclusions as those of Krivtsov and Morozov. ${ }^{18}$ On the other hand, Sun and Zhang ${ }^{21,22}$ presented a semicontinuum model for nanostructured materials and concluded that the Young's modulus of nanoplates is only two-thirds of the corresponding bulk value. Van Workum and de Pablo ${ }^{23}$ arrived at the same conclusion in a similar study based on the LennardJones potential. Nan et al. ${ }^{17}$ and Sharma and Ganti ${ }^{24}$ evaluated the dependence of the elastic properties of nanocrystalline materials on the grain size and obtained a lower elastic modulus of nanocrystalline materials. Villain et al. ${ }^{30}$ and Broughton and Meli ${ }^{29}$ also obtained lower elastic moduli of nanoscale materials than those of large-scale materials with direct atomistic simulation. Moreover, Villain et al. ${ }^{30}$ considered the effect of surface on the elastic properties. Miller and Shenoy ${ }^{26}$ constructed a simple model taking account of surface tension to predict the size-dependent elastic properties of nanoscale plates and beams and reached a conclusion that the effective stiffness is closely related to the values of surface elastic constants. Using a combination of molecular statics and $a b$ initio calculations, Zhou and Huang ${ }^{31}$ showed that elastic moduli can either increase or decrease at the 


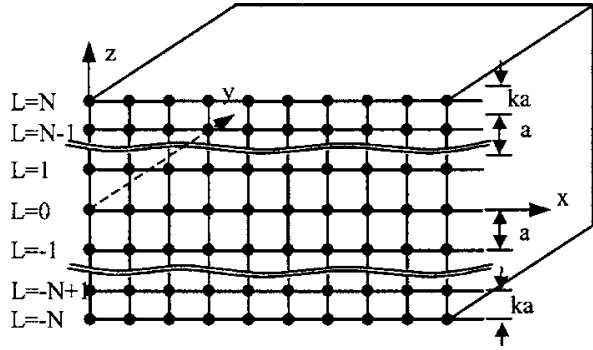

FIG. 1. The schematic of 3D model of a nanofilm

nanoscale, depending on the competition between atomic coordination and electron redistribution on the surface of nanocrystals.

As a matter of fact, the most elementary and the greatest difference between micro- and nanoscale materials and macroscopic materials is the increase of the surface-to-volume ratio. As a result, the surface effects become more important at the nanoscale, which greatly alter the mechanical properties of nanomaterials (strength, fracture toughness, elastic moduli, etc.). The effects of surface energy and surface tension on elastic properties were extensively discussed in the literature. ${ }^{8,19,25-27,30}$ Besides surface tension, the relaxation of surface plays an important role in the mechanical properties of nanomaterials. It is proposed that the coordination number imperfection of an atom at the surface of crystal causes the atom to relax spontaneously, and the bond of the atom will contract or expand. ${ }^{32,33}$ The relaxation can cause the variation of elastic properties of the surface of crystal, which may be insignificant at the macroscale, but will affect the elastic properties of the whole crystal when the dimension of crystal is at the nanoscale. In the present paper, a lattice model will be proposed to investigate the size-dependent properties of nanofilm, which takes the surface relaxation into consideration.

\section{3D MODEL OF THE NANOFILM THAT TAKES THE SURFACE RELAXATION INTO CONSIDERATION}

\section{A. The elastic constants of the 3D nanofilm model}

We first consider a three-dimensional simple cubic lattice model (shown in Fig. 1) of a nanofilm with uniform thickness $h$ and infinite length and width. Along the thickness, the model is assumed to have $2 N+1(N=1,2,3, \ldots$, layers of atoms, and a rectangular coordinate system is used with $x$ and $y$ axes in the middle surface and $z$ axis perpendicular to the surface (Fig. 1). The discrete solid dots denote atoms, and the short solid lines connecting these dots denote atomic bonds.

The surface relaxation is considered in the model. It is known that the termination of the lattice periodicity in the surface normal direction will lead to the imperfection of the coordination numbers (CNs) of a surface atom, which can make the remaining bonds of this lower-coordinated surface atom relax. The $\mathrm{CN}$-imperfection-induced bond relaxation can be defined as $a_{i}=k_{i} a,{ }^{33}$ where $a$ denotes the bulk value of bond length, the subscript $i$ is the $i$ th atomic layer, and $k_{i}$ is the relaxation coefficient. The relaxation may be in contraction $\left(k_{i}<1\right)$ or in expansion $\left(k_{i}>1\right)$. In addition, the degree

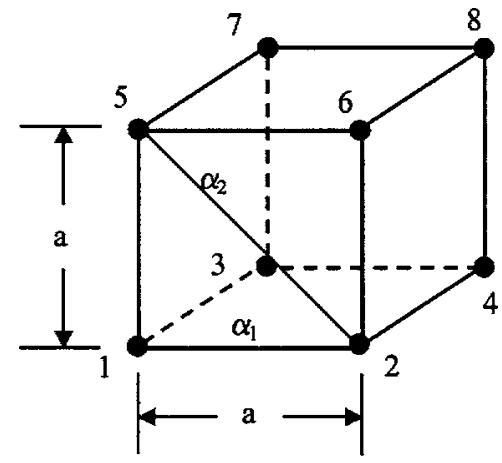

(a). Type A

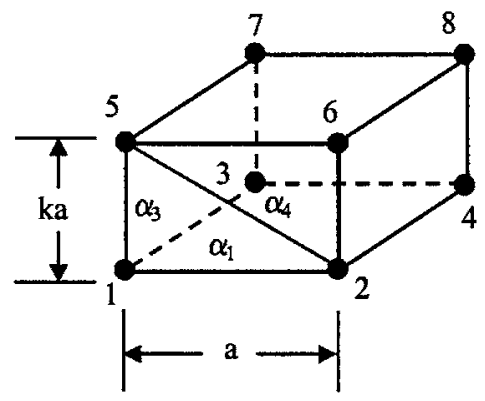

(b). Type B

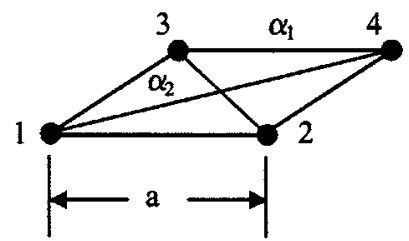

(c). Type C

FIG. 2. The schematics of three kinds of 3D typical lattice cells: (a) the element in the $j$ th layer $(-N<j<N-1)$, (b) the element in the $(N-1)$ th and the $-N$ th layer, and (c) the element in the most top (i.e., $N$ th) layer.

of the relaxation decays greatly along the inner-normal direction of the free surface, and no $\mathrm{CN}$ reduction is expected for $i>3$. Since our concern is only whether the surface relaxation has an influence on the elastic constants of the nanofilm or not, thus the relaxations of atoms at the sub and third outmost layers can be neglected for simplification and without loss of generality. Consequently, in the three-dimensional (3D) lattice model, the atomic layers between the $-(N$ $-1)$ th and the $(N-1)$ th layers are uniform with a constant lattice distance $a$, while for atoms at the top and the bottom layers, their distance with the nearest atomic layer will be specified as $k a$, where $k<1$ if the relaxation is in contraction and $k>1$ if the relaxation is in expansion.

Each atom interacts with its nearest- and next-nearest neighbors and the interactions are, respectively, represented by springs with spring constants $\alpha_{1}, \alpha_{2}, \alpha_{3}$, and $\alpha_{4}$ (as shown in Fig. 2). If we calculate the deformation energy of the nanostructure from the left-bottom atom and atom by atom, layer by layer, then there are three kinds of typical representative cells (as shown in Fig. 2). Type $A$ represents the cell in the layers from the $-(N-1)$ th to the $(N-2)$ th layers, type $B$ represents the cell at the $(-N)$ th layer and the $(N-1)$ th layer, 
and type $C$ is the cell at the top layer. In the cells of types $A$ and $C$, the spring constants of the nearest neighbors is $\alpha_{1}$, and the spring constants of the next-nearest neighbors is $\alpha_{2}$. In the cells of type $B$, the spring constants of the nearest neighbors are $\alpha_{1}$ and $\alpha_{3}$, respectively, and the spring constants of the next-nearest neighbors are $\alpha_{2}$ and $\alpha_{4}$, respectively. The deformation energies of the three types of cells are, respectively, expressed as $U_{(i j k)}^{A}, U_{(i j k)}^{B}$, and $U_{(i j N)}^{C}$, where the subscript $(i j k)$ denotes the left-bottom atom of the cell. Then the total deformation energy of the nanofilm can be obtained by the sum of all cells

$$
\begin{aligned}
U_{\text {tot }}= & \sum_{i} \sum_{j} \sum_{k}^{-(N-1) \leqslant k \leqslant(N-2)} U_{(i j k)}^{A}+\sum_{i} \sum_{j} \sum_{k}^{2} U_{(i j k)}^{B} \\
& +\sum_{i} \sum_{j} U_{(i j N)}^{C} .
\end{aligned}
$$

In the expression above, the contribution of energy from the rightmost and the backmost faces is neglected due to small thickness.

In three types of cells, the number 1 atom occupies the position of the $i$ th, $j$ th rows, and $k$ th column. Then the coordinates of the atoms in the cell are (1) $\left(x_{i}, y_{j}, z_{k}\right),(2)$ $\left(x_{i+1}, y_{j}, z_{k}\right)$, (3) $\left(x_{i}, y_{j+1}, z_{k}\right)$, (4) $\left(x_{i+1}, y_{j+1}, z_{k}\right)$, $\left(x_{i}, y_{j}, z_{k+1}\right)$, (6) $\left(x_{i+1}, y_{j}, z_{k+1}\right)$, (7) $\left(x_{i}, y_{j+1}, z_{k+1}\right)$, and $\left(x_{i+1}, y_{j+1}, z_{k+1}\right)$.

For the nanofilm, the dimension in the thickness direction is in nanoscale and can finally be reduced to several layers of atoms in our discussion, which are only a few nanometers. So the physical quantities in the $z$ direction are discrete, where a discrete treatment is applied. However, the dimensions of the nanofilm in the $x$ and $y$ directions are not in nanoscale and are very large relative to its thickness dimension. Therefore, although the lattice model is also used in the two directions, the physical quantities are continuous as a whole. So the classical continuum treatment can be employed in the $x$ and $y$ directions. In other words, the physical quantities are viewed as continuous in each layer but discrete among the layers. The displacements of the atom $\left(x_{i}, y_{j}, z_{k}\right)$ in the $k$ th layer are expressed as $u_{k}\left(x_{i}, y_{j}\right), v_{k}\left(x_{i}, y_{j}\right)$, and $w_{k}\left(x_{i}, y_{j}\right)$, respectively.

The deformation energy of three types of representative cells can be, respectively, written as

$$
\begin{aligned}
U_{(i j k)}^{m}= & U_{1-2}^{m}+U_{1-3}^{m}+U_{1-4}^{m}+U_{2-3}^{m}+U_{1-5}^{m}+U_{1-6}^{m}+U_{1-7}^{m} \\
& +U_{2-5}^{m}+U_{3-5}^{m} \quad(m=A, B), \\
U_{(i j N)}^{C}= & U_{1-2}^{C}+U_{1-3}^{C}+U_{1-4}^{C}+U_{2-3}^{C},
\end{aligned}
$$

where $U_{i-j}^{m}$ denotes the deformation energy of the type $m$ ( $m=A, B$, and $C$ ) cell due to the stretching of the spring between atoms $i$ and $j$. The atomic pairs, of which the interactions are not included in Eqs. (2) and (3), are considered in the nearest cell.

In what follows, we will calculate the deformation energy of every pair of atoms, respectively. Since the elastic constants considered here are effective or average values of the elastic constants of the nanofilm, it is assumed that the strains in the nanofilm be uniform. Deformation energy $U_{1-2}^{m}$
( $m=A, B$, and $C$ ) is due to the stretching of the nearestneighbor spring between atoms 1 and 2. The displacement of atom 2 can be expanded ${ }^{21}$ in a Taylor series with two terms as

$$
u_{k}\left(x_{i+1}, y_{j}\right)=u_{k}\left(x_{i}, y_{j}\right)+\left.\frac{\partial u_{k}(x, y)}{\partial x}\right|_{\left(x_{i}, y_{j}\right)}\left(x_{i+1}-x_{i}\right)
$$

Since $x_{i+1}-x_{i}=a$, the deformation energy $U_{1-2}^{m}(m=A, B$, and $C)$ can be expressed ${ }^{21}$ in the following form:

$$
\begin{aligned}
U_{1-2}^{m} & =\frac{1}{2} \alpha_{1}\left[u_{k}\left(x_{i+1}, y_{j}\right)-u_{k}\left(x_{i}, y_{j}\right)\right]^{2} \\
& \approx \frac{1}{2} \alpha_{1} a^{2}\left[\left.\frac{\partial u_{k}(x, y)}{\partial x}\right|_{\left(x_{i}, y_{j}\right)}\right]^{2} .
\end{aligned}
$$

In the same way, the deformation energies $U_{1-3}^{m}(m=A$, $B$, and $C$ ) and $U_{1-5}^{A}$ can be expressed in the following forms, respectively:

$$
\begin{aligned}
U_{1-3}^{m} & =\frac{1}{2} \alpha_{1}\left[v_{k}\left(x_{i}, y_{j+1}\right)-v_{k}\left(x_{i}, y_{j}\right)\right]^{2} \\
& \approx \frac{1}{2} \alpha_{1} a^{2}\left[\left.\frac{\partial v_{k}(x, y)}{\partial y}\right|_{\left(x_{i}, y_{j}\right)}\right]^{2}, \\
U_{1-5}^{A} & =\frac{1}{2} \alpha_{1}\left[w_{k+1}\left(x_{i}, y_{j}\right)-w_{k}\left(x_{i}, y_{j}\right)\right]^{2} \\
& \approx \frac{1}{2} \alpha_{1} a^{2}\left[\left.\frac{w_{k+1}-w_{k}}{z_{k+1}-z_{k}}\right|_{\left(x_{i}, y_{j}\right)}\right]^{2} .
\end{aligned}
$$

However, for the cells of type $B, z_{k+1}-z_{k}=k a$, the deformation energy $U_{1-5}^{B}$ takes the following form:

$$
U_{1-5}^{B} \approx \frac{1}{2} \alpha_{3} k^{2} a^{2}\left[\left.\frac{w_{k+1}-w_{k}}{z_{k+1}-z_{k}}\right|_{\left(x_{i}, y_{j}\right)}\right]^{2} \text {. }
$$

The stretching of the next-nearest spring between atoms 1 and 4 in each cell is ${ }^{21}$

$$
\begin{aligned}
\sqrt{[a} & \left.+u_{k}\left(x_{i+1}, y_{j+1}\right)-u_{k}\left(x_{i}, y_{j}\right)\right]^{2}+\left[a+v_{k}\left(x_{i+1}, y_{j+1}\right)-v_{k}\left(x_{i}, y_{j}\right)\right]^{2} \\
& -\sqrt{2} a .
\end{aligned}
$$

By using the binomial expansion, the above expression can be approximated by

$$
\frac{\sqrt{2}}{2}\left\{\left[u_{k}\left(x_{i+1}, y_{j+1}\right)-u_{k}\left(x_{i}, y_{j}\right)\right]+\left[v_{k}\left(x_{i+1}, y_{j+1}\right)-v_{k}\left(x_{i}, y_{j}\right)\right]\right\} .
$$

The displacements $u_{k}\left(x_{i+1}, y_{j+1}\right)$ and $v_{k}\left(x_{i+1}, y_{j+1}\right)$ of atom 4 can be similarly expanded in a Taylor series with two terms at the point $\left(x_{i}, y_{j}, z_{k}\right)$. Therefore, the deformation energy $U_{1-4}^{m}(m=A, B$, and $C)$ due to the stretching of the spring between atoms 1 and 4 in three types of cells is obtained as 


$$
\begin{aligned}
U_{1-4}^{m}= & \frac{1}{4} \alpha_{2} a^{2}\left\{\left.\left[\frac{\partial u_{k}(x, y)}{\partial x}+\frac{\partial v_{k}(x, y)}{\partial y}\right]\right|_{\left(x_{i}, y_{j}\right)}\right. \\
& \left.+\left.\left[\frac{\partial u_{k}(x, y)}{\partial y}+\frac{\partial v_{k}(x, y)}{\partial x}\right]\right|_{\left(x_{i}, y_{j}\right)}\right\}^{2}
\end{aligned}
$$

Similarly, the deformation energy $U_{2-3}^{m}(m=A, B$, and $C)$ due to the stretching of the spring between atoms 2 and 3 in three types of cells is written as

$$
\begin{aligned}
U_{2-3}^{m}= & \frac{1}{4} \alpha_{2} a^{2}\left\{\left.\left[\frac{\partial u_{k}(x, y)}{\partial x}+\frac{\partial v_{k}(x, y)}{\partial y}\right]\right|_{\left(x_{i}, y_{j}\right)}\right. \\
& \left.-\left.\left[\frac{\partial u_{k}(x, y)}{\partial y}+\frac{\partial v_{k}(x, y)}{\partial x}\right]\right|_{\left(x_{i}, y_{j}\right)}\right\}^{2} .
\end{aligned}
$$

Thus,

$$
\begin{aligned}
U_{1-4}^{m}+U_{2-3}^{m}= & \frac{1}{2} \alpha_{2} a^{2}\left\{\left.\left[\frac{\partial u_{k}(x, y)}{\partial x}+\frac{\partial v_{k}(x, y)}{\partial y}\right]^{2}\right|_{\left(x_{i}, y_{j}\right)}\right. \\
& \left.-\left.\left[\frac{\partial u_{k}(x, y)}{\partial y}+\frac{\partial v_{k}(x, y)}{\partial x}\right]^{2}\right|_{\left(x_{i}, y_{j}\right)}\right\} .
\end{aligned}
$$

In a similar way, for a cell of type $A$, we have

$$
\begin{aligned}
U_{1-6}^{m}+U_{2-5}^{m}= & \frac{1}{2} \alpha_{2} a^{2}\left\{\left.\left[\frac{\partial u_{k}(x, y)}{\partial x}+\frac{w_{k+1}-w_{k}}{z_{k+1}-z_{k}}\right]^{2}\right|_{\left(x_{i}, y_{j}\right)}\right. \\
& \left.-\left.\left[\frac{u_{k+1}-u_{k}}{z_{k+1}-z_{k}}+\frac{\partial w_{k}(x, y)}{\partial x}\right]^{2}\right|_{\left(x_{i}, y_{j}\right)}\right\}, \quad(14) \\
U_{1-7}^{m}+U_{3-5}^{m}= & \frac{1}{2} \alpha_{2} a^{2}\left\{\left.\left[\frac{\partial v_{k}(x, y)}{\partial y}+\frac{w_{k+1}-w_{k}}{z_{k+1}-z_{k}}\right]^{2}\right|_{\left(x_{i}, y_{j}\right)}\right. \\
& \left.-\left.\left[\frac{v_{k+1}-v_{k}}{z_{k+1}-z_{k}}+\frac{\partial w_{k}(x, y)}{\partial y}\right]^{2}\right|_{\left(x_{i}, y_{j}\right)}\right\} .
\end{aligned}
$$

On the other hand, for a cell of type $B, y_{j+1}-y_{j}=k a$, the stretching of the next-nearest neighbor spring between atoms 1 and 6 becomes

$$
\begin{aligned}
& \sqrt{\left[a+u_{k+1}\left(x_{i+1}, y_{j}\right)-u_{k}\left(x_{i}, y_{j}\right)\right]^{2}+\left[k a+w_{k+1}\left(x_{i+1}, y_{j}\right)-w_{k}\left(x_{i}, y_{j}\right)\right]^{2}} \\
& \quad-\sqrt{a^{2}+(k a)^{2}} .
\end{aligned}
$$

After the binomial expansion, the expression above can be approximated by

$$
\begin{aligned}
& \frac{1}{\sqrt{1+k^{2}}}\left\{\left[u_{k+1}\left(x_{i+1}, y_{j}\right)-u_{k}\left(x_{i}, y_{j}\right)\right]+k\left[w_{k+1}\left(x_{i+1}, y_{j}\right)\right.\right. \\
& \left.\left.-w_{k}\left(x_{i}, y_{j}\right)\right]\right\} .
\end{aligned}
$$

In the same way, the displacements $u_{k+1}\left(x_{i+1}, y_{j}\right)$ and $v_{k+1}\left(x_{i+1}, y_{j}\right)$ of atom 6 can be, respectively, expanded in a Taylor series with two terms at the point $\left(x_{i}, y_{j}, z_{k}\right)$. Then the deformation energy $U_{1-6}^{B}$ due to the stretching of the spring between atoms 1 and 6 in the cell of type $B$ is obtained as

$$
\begin{aligned}
U_{1-6}^{B}= & \frac{1}{2} \frac{\alpha_{4} a^{2}}{1+k^{2}}\left\{\left.\left[\frac{\partial u_{k}(x, y)}{\partial x}+k^{2} \frac{w_{k+1}-w_{k}}{z_{k+1}-z_{k}}\right]\right|_{\left(x_{i}, y_{j}\right)}\right. \\
& \left.+\left.k\left[\frac{u_{k+1}-u_{k}}{z_{k+1}-z_{k}}+\frac{\partial w(x, y)}{\partial x}\right]\right|_{\left(x_{i}, y_{j}\right)}\right\}^{2} .
\end{aligned}
$$

The deformation energies $U_{2-5}^{B}, U_{1-7}^{B}$ and $U_{3-5}^{B}$ can be, respectively, derived in the similar form. Thus, the following expressions can be obtained for the cell of type $B$ :

$$
\begin{aligned}
U_{1-6}^{B}+U_{2-5}^{B}= & \frac{\alpha_{4} a^{2}}{1+k^{2}}\left\{\left.\left[\frac{\partial u(x, y)}{\partial x}+k^{2} \frac{w_{k+1}-w_{k}}{z_{k+1}-z_{k}}\right]^{2}\right|_{\left(x_{i}, y_{j}\right)}\right. \\
& \left.+\left.k^{2}\left[\frac{u_{k+1}-u_{k}}{z_{k+1}-z_{k}}+\frac{\partial w(x, y)}{\partial x}\right]^{2}\right|_{\left(x_{i}, y_{j}\right)}\right\}, \\
U_{1-7}^{B}+U_{3-5}^{B}= & \frac{\alpha_{4} a^{2}}{1+k^{2}}\left\{\left.\left[\frac{\partial v(x, y)}{\partial y}+k^{2} \frac{w_{k+1}-w_{k}}{z_{k+1}-z_{k}}\right]^{2}\right|_{\left(x_{i}, y_{j}\right)}\right. \\
& \left.+\left.k^{2}\left[\frac{v_{k+1}-v_{k}}{z_{k+1}-z_{k}}+\frac{\partial w(x, y)}{\partial y}\right]^{2}\right|_{\left(x_{i}, y_{j}\right)}\right\} .
\end{aligned}
$$

Assuming that the deformation is small, we can define the elastic strains in the nanofilm as

$$
\begin{aligned}
& \varepsilon_{x}=\frac{\partial u}{\partial x}, \quad \varepsilon_{y}=\frac{\partial v}{\partial y}, \quad \varepsilon_{z}=\frac{w_{k+1}-w_{k}}{z_{k+1}-z_{k}}, \\
& \varepsilon_{x y}=\frac{1}{2}\left(\frac{\partial u}{\partial y}+\frac{\partial v}{\partial x}\right), \quad \varepsilon_{y z}=\frac{1}{2}\left(\frac{v_{k+1}-v_{k}}{z_{k+1}-z_{k}}+\frac{\partial w}{\partial y}\right), \\
& \varepsilon_{x z}=\frac{1}{2}\left(\frac{u_{k+1}-u_{k}}{z_{k+1}-z_{k}}+\frac{\partial w}{\partial x}\right) .
\end{aligned}
$$

Substituting Eqs. (21) into Eqs. (5)-(8), (13)-(15), (19), and (20), respectively, the deformation energy of every pair of atoms in the nanofilm can be expressed as the functions of strain. Furthermore, substituting these equations into Eqs. (2) and (3), the deformation energy of three kinds of representative cells can be written as

$$
\begin{aligned}
U_{(i j k)}^{A}= & \frac{a^{2}}{2}\left\{\alpha_{1}\left(\varepsilon_{x}^{2}+\varepsilon_{y}^{2}+\varepsilon_{z}^{2}\right)+\alpha_{2}\left[\left(\varepsilon_{x}+\varepsilon_{y}\right)^{2}+\left(\varepsilon_{x}+\varepsilon_{z}\right)^{2}\right.\right. \\
& \left.\left.+\left(\varepsilon_{y}+\varepsilon_{z}\right)^{2}\right]+4 \alpha_{2}\left(\varepsilon_{x y}^{2}+\varepsilon_{y z}^{2}+\varepsilon_{x z}^{2}\right)\right\}, \\
U_{(i j k)}^{B}= & \frac{a^{2}}{2}\left\{\alpha_{1}\left(\varepsilon_{x}^{2}+\varepsilon_{y}^{2}\right)+k^{2} \alpha_{3} \varepsilon_{z}^{2}+\alpha_{2}\left[\left(\varepsilon_{x}+\varepsilon_{y}\right)^{2}+4 \varepsilon_{x y}^{2}\right]\right\} \\
& +\frac{\alpha_{4} a^{2}}{1+k^{2}}\left\{\left[\left(\varepsilon_{x}+k^{2} \varepsilon_{z}\right)^{2}+4 k^{2} \varepsilon_{x z}^{2}\right]+\left[\left(\varepsilon_{y}+k^{2} \varepsilon_{z}\right)^{2}\right.\right. \\
& \left.\left.+4 k^{2} \varepsilon_{y z}^{2}\right]\right\}, \\
U_{(i j N)}^{C}= & \frac{a^{2}}{2}\left\{\alpha_{1}\left(\varepsilon_{x}^{2}+\varepsilon_{y}^{2}\right)+\alpha_{2}\left[\left(\varepsilon_{x}+\varepsilon_{y}\right)^{2}+4 \varepsilon_{x y}^{2}\right]\right\} .
\end{aligned}
$$

Obviously, the total strain energy of the nanofilm can be obtained by substituting Eqs. (22)-(24) into Eq. (1). The av- 
erage strain energy density is calculated via the ratio between the total strain energy $U_{\text {tot }}$ and the volume $V_{0}$ of the nanofilm, i.e.,

$$
f=\frac{U_{\text {tot }}}{V_{0}} .
$$

Because the cells are uniform in the $x-y$ plane, we may choose a crystal lattice width in either direction for the cell, i.e., $V_{0}=a \times a \times h$. Here, $h$ is the thickness of the nanofilm. The value of $h$ cannot be determined unambiguously. ${ }^{18}$ The spacing between the outermost layer and the next layer is $k a$, and that between other two neighboring layers is $a$ (as shown in Fig. 1). The thickness of a single-atom layer is defined to be equal to the spacing between the layer and its neighbor layer. If the thickness $h$ is defined as the sum of the spacing between the atomic layers of the crystal, then $h=2(N+k$ -1) $a$ since the nanofilm has $2 N+1$ layers. On the other hand, it is reasonable to define the crystal thickness as the sum of the thickness of atomic layers in the nanofilm, i.e., $h=[2(N$ $+k)-1] a$. For this reason, an ambiguous definition of the thickness $h$ can be introduced as

$$
h=[2(N+k)-1-\beta] a \quad(0 \leqslant \beta \leqslant 1),
$$

where $\beta$ is the parameter of ambiguity. Obviously, $h=2(N$ $+k-1) a$ if $\beta=1$, and $h=[2(N+k)-1] a$ if $\beta=0$.

The strain energy density function $f$ of the volume $V_{0}$ can be obtained as

$$
\begin{aligned}
f= & \frac{1}{[2(N+k)-1-\beta] a}\left\{(N-1) \alpha_{1}\left(\varepsilon_{x}^{2}+\varepsilon_{y}^{2}+\varepsilon_{z}^{2}\right)\right. \\
& +\frac{3 \alpha_{1}}{2}\left(\varepsilon_{x}^{2}+\varepsilon_{y}^{2}\right)+k^{2} \alpha_{3} \varepsilon_{z}^{2}+\frac{3 \alpha_{2}}{2}\left(\varepsilon_{x}+\varepsilon_{y}\right)^{2} \\
& +(N-1) \alpha_{2}\left[\left(\varepsilon_{x}+\varepsilon_{y}\right)^{2}+\left(\varepsilon_{y}+\varepsilon_{z}\right)^{2}+\left(\varepsilon_{x}+\varepsilon_{z}\right)^{2}\right] \\
& +\frac{2 \alpha_{4}}{1+k^{2}}\left[\left(\varepsilon_{x}+k^{2} \varepsilon_{z}\right)^{2}+\left(\varepsilon_{y}+k^{2} \varepsilon_{z}\right)^{2}\right]+2(2 N+1) \alpha_{2} \varepsilon_{x y}^{2} \\
& +\left[4(N-1) \alpha_{2}+\frac{8 k^{2} \alpha_{4}}{1+k^{2}}\right] \varepsilon_{x z}^{2} \\
& \left.+\left[4(N-1) \alpha_{2}+\frac{8 k^{2} \alpha_{4}}{1+k^{2}}\right] \varepsilon_{y z}^{2}\right\} .
\end{aligned}
$$

Since $\sigma_{i j}=\partial f / \partial \varepsilon_{i j}$, the constitutive relation of the nanofilm can be expressed as

$$
\sigma_{i}=C_{i j} \varepsilon_{j} \quad(i, j=1,2, \ldots, 6),
$$

where subscript $i=1,2, \ldots, 6$ corresponds to $x, y, z, x y, y z$, and $x z$, respectively. $C_{i j}$ gives 36 elastic constants, in which nonzero constants are

$$
\begin{aligned}
& C_{11}=C_{22}=\frac{(2 N+1) \alpha_{1}+(4 N-1) \alpha_{2}+\left(4 \alpha_{4} / 1+k^{2}\right)}{[2(N+k)-1-\beta] a}, \\
& C_{12}=C_{21}=\frac{(2 N+1) \alpha_{2}}{[2(N+k)-1-\beta] a}, \\
& C_{13}=C_{31}=C_{23}=C_{32}=\frac{2(N-1) \alpha_{2}+\left(4 k^{2} \alpha_{4} / 1+k^{2}\right)}{[2(N+k)-1-\beta] a},
\end{aligned}
$$

$C_{33}=\frac{2(N-1)\left(\alpha_{1}+2 \alpha_{2}\right)+2 k^{2} \alpha_{3}+\left(8 k^{4} \alpha_{4} / 1+k^{2}\right)}{[2(N+k)-1-\beta] a}$,

$C_{44}=4 C_{12}, \quad C_{55}=C_{66}=4 C_{13}$.

It is obvious that there are four independent elastic constants, i.e., $C_{11}, C_{12}, C_{13}$, and $C_{33}$. The nanofilm is not isotropic due to surface relaxation. It can be shown from the descriptions above that the elastic constants are dependent on the layer number $N$ and the relaxation coefficient $k$. The dependence will be analyzed in Sec. II B.

We introduce another four elastic moduli as

$$
\begin{aligned}
& E_{1}=\frac{\left(C_{11}-C_{12}\right)\left(C_{11} C_{33}+C_{12} C_{33}-2 C_{13}^{2}\right)}{C_{11} C_{33}-C_{13}^{2}}, \\
& \nu_{1}=C_{12} C_{33}-C_{13}^{2} / C_{11} C_{33}-C_{13}^{2}, \\
& E_{2}=\frac{C_{11} C_{33}+C_{12} C_{33}-2 C_{13}^{2}}{C_{11}+C_{12}}, \quad \nu_{2}=\frac{C_{13}}{C_{11}+C_{12}} .
\end{aligned}
$$

Then the constitutive relation of the nanofilm can also be written as

$$
\begin{aligned}
& \varepsilon_{x}=\frac{1}{E_{1}}\left(\sigma_{x}-\nu_{1} \sigma_{y}\right)-\frac{\nu_{2}}{E_{2}} \sigma_{z}, \\
& \varepsilon_{y}=\frac{1}{E_{1}}\left(\sigma_{y}-\nu_{1} \sigma_{x}\right)-\frac{\nu_{2}}{E_{2}} \sigma_{z}, \\
& \varepsilon_{z}=\frac{1}{E_{2}} \sigma_{z}-\frac{\nu_{2}}{E_{2}}\left(\sigma_{x}+\sigma_{y}\right), \\
& \varepsilon_{x y}=\frac{\sigma_{x y}}{2 G_{1}}, \quad \varepsilon_{y z}=\frac{\sigma_{y z}}{2 G_{2}}, \quad \varepsilon_{x z}=\frac{\sigma_{x z}}{2 G_{2}},
\end{aligned}
$$

where shear moduli $G_{1}$ and $G_{2}$ are not independent and can be expressed by the four elastic constants given above, i.e.,

$$
\begin{aligned}
& G_{1}=\frac{C_{44}}{2}=\frac{2 E_{1}\left(E_{1} \nu_{2}^{2}-E_{2} \nu_{1}\right)}{\left(1+\nu_{1}\right)\left[2 E_{1} \nu_{2}^{2}-E_{2}\left(1-\nu_{1}\right)\right]}, \\
& G_{2}=\frac{C_{55}}{2}=\frac{2 E_{1} E_{2} \nu_{2}}{2 E_{1} \nu_{2}^{2}-E_{2}\left(1-\nu_{1}\right)} .
\end{aligned}
$$

\section{B. A discussion about the 3D elastic constants}

It can be found from the above expressions that the elastic constants $C_{11}, C_{12}, C_{13}$, and $C_{33}$ are dependent on the thickness of the nanofilm and the relaxation on its surface. The thickness of the nanofilm contains an ambiguity parameter $\beta$. The relaxation coefficient also varies with different crystals and different crystal orientations. It is smaller than 1 when the relaxation is in contraction and greater than 1 when the relaxation is in expansion. For example, the expansion coefficients of $\mathrm{Be}, \mathrm{Mg}, \mathrm{Hg}$, and $\mathrm{Zn}$ at the hcp(0001) surface are $1.11,1.22,1.21$, and $1.50,{ }^{32}$ respectively, and the contraction coefficients of $\mathrm{Zr}, \mathrm{Fe}$, and $\mathrm{Ag}$ at the hcp(0001) surface are $0.71,0.81$, and $0.88,{ }^{32}$ respectively. 


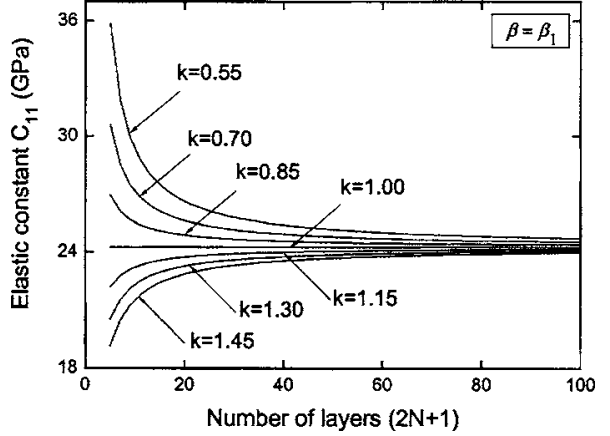

FIG. 3. The variations of elastic constant $C_{11}$ with the number of atomic layers with respect to different values of relaxation coefficient when the thickness parameter $\beta=\beta_{1}$.

When the parameter $\beta$ and the relaxation coefficient $k$ are specified, it can be determined whether the four elastic constants will increase or decrease with the decrease of atomic layer numbers. For a certain value of $\beta$, the critical value $k_{c}$ of $k$ can be defined as the one when one of the elastic constants keeps constant to the variations of atomic layer numbers. The elastic constant increases or decreases with the decrease of atomic layer numbers when $k<k_{c}$ or $k>k_{c}$. In the same way, if the $k_{c}$ is specified, the characteristic value of $\beta$ can also be similarly defined. It is reasonable to let $k_{c}=1$, and then the three characteristic values of $\beta$, which make elastic constants $C_{11}, C_{12}, C_{13}$, and $C_{33}$ size independent, respectively, can be calculated as

$$
\beta_{1}=\frac{\alpha_{1}}{\alpha_{1}+2 \alpha_{2}}, \quad \beta_{2}=0, \quad \beta_{3}=1
$$

where $\beta_{2}<\beta_{1}<\beta_{3}$, and the thickness $h$ of the nanofilm reaches minimum as $\beta=\beta_{3}$, which is adopted by Krivtsov and Morozov, ${ }^{18}$ and $h$ reaches maximum as $\beta=\beta_{2}$, which is adopted by Sun and Zhang. ${ }^{21}$ When the relaxation coefficient $k=k_{c}=1$, the elastic constant $C_{11}$ is size independent for $\beta$ $=\beta_{1}, C_{12}$ is size independent for $\beta=\beta_{2}$, and $C_{13}$ and $C_{33}$ are size independent for $\beta=\beta_{3}$. When the relaxation coefficient $k \neq k_{c}$, elastic constants are size dependent, as will be discussed in detail.

The spring constants $\alpha_{1}$ and $\alpha_{2}$ in the calculation can be obtained by the lattice dynamics method. We define, respectively, spring constants $\alpha_{3}$ and $\alpha_{4}$ as

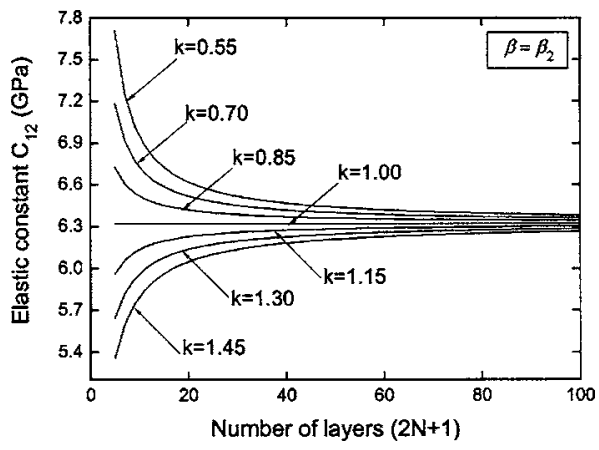

FIG. 4. The variations of elastic constant $C_{12}$ with the number of atomic layers with respect to different values of relaxation coefficient when the thickness parameter $\beta=\beta_{2}$.

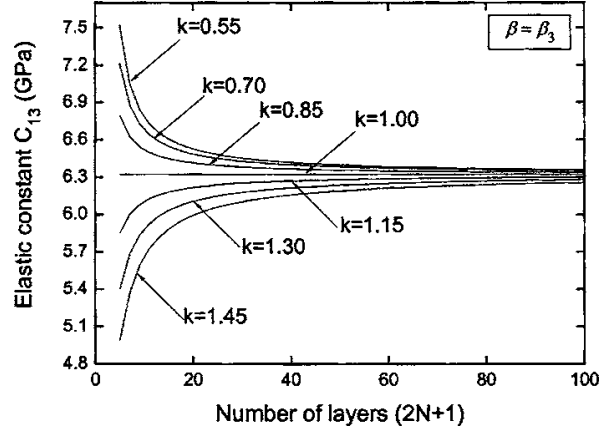

FIG. 5. The variations of elastic constant $C_{13}$ with the number of atomic layers with respect to different values of relaxation coefficient when the thickness parameter $\beta=\beta_{3}$.

$$
\alpha_{3}=k^{-n} \alpha_{1}, \quad \alpha_{4}=k^{-n} \alpha_{2} \quad(n=1,2,3, \ldots,) .
$$

As a matter of fact, the effect of the value $n$ is negligible, so we can choose $n=1$ for the simplification of calculation, i.e., $\alpha_{3}=\alpha_{1} / k$ and $\alpha_{4}=\alpha_{2} / k$. The values of parameters used in the calculation are $a=1.74 \times 10^{-10} \mathrm{~m}, \alpha_{1}=2.02 \mathrm{~N} / \mathrm{m}$, and $\alpha_{2}$ $=1.10 \mathrm{~N} / \mathrm{m},{ }^{21}$ respectively.

Figures 3-6 show the variations of elastic constants with the numbers of atomic layers for different relaxation coefficients $k$ and characteristic parameters $\beta$. It is found that the elastic constants $C_{11}, C_{12}, C_{13}$, and $C_{33}$ are all size dependent when the relaxation coefficient $k \neq k_{c}$. When the atomic layer numbers are less than about 50, the elastic constants vary with the decrease of the atomic layer number. They vary sharply as the atomic layer numbers are less than 20. On the other hand, the elastic constants approach constant values as the number of atom layers tends to infinity. In addition, the elastic constants are related with the surface relaxation. Figure 3 shows the variations of elastic constant $C_{11}$ with the decrease of atomic layer numbers when the parameter $\beta$ $=\beta_{1}$. It can be found that the elastic constant $C_{11}$ decreases with the decrease of the atomic layer numbers when the relaxation coefficient is greater than the critical value $k_{c}$, which corresponds to the case of surface expanding. On the other hand, the elastic constant increases when the relaxation coefficient is smaller than the critical value, which corresponds to the case of surface contraction. The smaller the relaxation coefficient $k$ is, the greater the value of elastic constant $C_{11}$ is. When the atomic layer number is 5 , the value of $C_{11}$ increases by $47.8 \%$ of the macroscopic value for $k=0.55$,

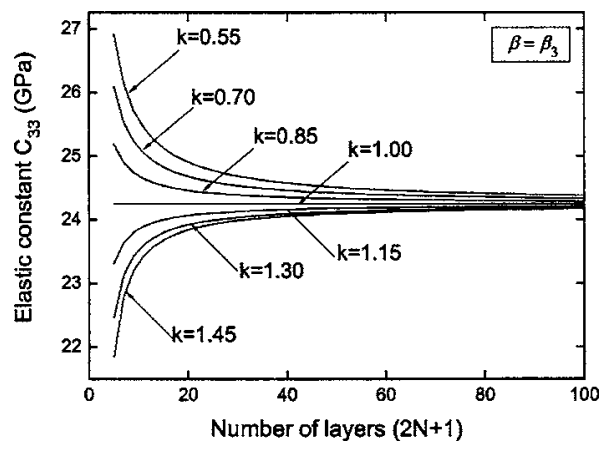

FIG. 6. The variations of elastic constant $C_{33}$ with the number of atomic layers with respect to different values of relaxation coefficient when the thickness parameter $\beta=\beta_{3}$. 


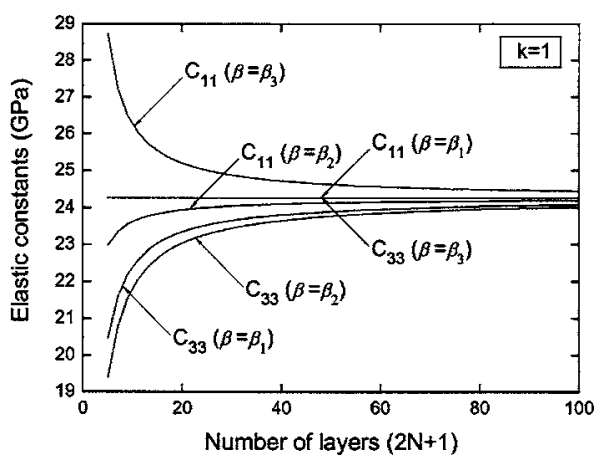

FIG. 7. The variations of elastic constants $C_{11}$ and $C_{33}$ with the number of the atomic layers with respect to different values of $\beta$ when the relaxation coefficient $k=1$.

and it decreases by $21.1 \%$ of the macroscopic value for $k$ $=1.45$. Figure 4 shows the variations of elastic constant $C_{12}$ with the decrease of atomic layer numbers when the parameter $\beta=\beta_{2}$, and similar results are obtained. When the atomic layer number is 5 , the value of $C_{12}$ increases by $22.0 \%$ of the macroscopic value for $k=0.55$, and it decreases by $15.3 \%$ of the macroscopic value for $k=1.45$. Figures 5 and 6 show the variations of elastic constants $C_{13}$ and $C_{33}$ with the decrease of atomic layer numbers when the parameter $\beta=\beta_{3}$. It can be seen that elastic constant $C_{13}$ decreases but $C_{33}$ increases with the decrease of the atomic layer numbers when the relaxation coefficient is greater than the critical value $k_{c}$. On the other hand, the elastic constant $C_{13}$ increases but $C_{33}$ decreases when the relaxation coefficient is smaller than the critical value. When the atomic layer number is 5 , the value of $C_{13}$ increases by $19.0 \%$ of the macroscopic value and the value of $C_{33}$ decreases by $10.0 \%$ for $k=0.55$, and the value of $C_{13}$ decreases by $21.0 \%$ of the macroscopic value and the value of $C_{33}$ increases by $11.0 \%$ for $k=1.45$.

The definition of the thickness of the nanofilm $h$ also has an important influence on the size effect of elastic constants, which is expressed via the parameter $\beta$. Figures 7 and 8 show the variations of elastic constants with respect to different characteristic values of $\beta$ when the surface relaxation is neglected, i.e., $k=1$. When $\beta$ is, respectively, equal to $\beta_{1}$, $\beta_{2}$, and $\beta_{3}$, the elastic constant $C_{11}$ keeps constant, decreases, and increases with the decrease of atomic layer numbers accordingly. $C_{12}$ keeps constant if $\beta=\beta_{2}$ and increases if $\beta$ is equal to $\beta_{1}$ or $\beta_{3}$. $C_{13}$ and $C_{33}$ keep constant if $\beta=\beta_{3}$

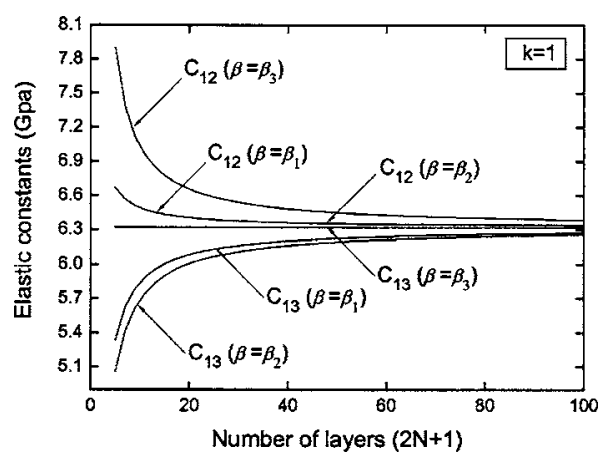

FIG. 8. The variations of elastic constants $C_{12}$ and $C_{13}$ with the number of the atomic layers with respect to different values of $\beta$ when the relaxation coefficient $k=1$. and decrease if $\beta$ is equal to $\beta_{1}$ or $\beta_{2}$. These results are summarized in Table I, where the sign "-_" denotes the size independence of the elastic constants, " $\uparrow "$ denotes the increase of the elastic constants, " $\downarrow$ " denotes the decrease of the elastic constants, and "/" denotes uncertain values.

As the number of atomic layer tends to infinity, it can be seen from Figs. 7 and 8 that the elastic constants $C_{11}, C_{33}$, $C_{12}$, and $C_{13}$, respectively, tend to certain values, i.e., their macroscopic counterparts, which are $C_{11}=C_{33}=24.3 \mathrm{GPa}$ and $C_{12}=C_{13}=6.3 \mathrm{GPa}$ for the present case. Therefore, the anisotropic nanofilm goes back to an isotropic material when the scale of material is macroscopic, and the elastic constants are no longer dependent on size and surface relaxation.

\section{PLANE PROBLEM OF THE NANOFILM}

In Sec. II B, four elastic constants of the 3D nanofilm model are obtained. In some cases, simplifications can be introduced, and the $3 \mathrm{D}$ problem can be reduced into a $2 \mathrm{D}$ plane problem. There are two kinds of plane problems in elasticity, plane stress and plane strain. If the length (in $y$ direction) and width (in $x$ direction) of the nanofilm are of the same quantitative order, the problem can be treated as in the plane-stress state when the uniform tension or pressure is exerted in the length or width direction. Here, $\sigma_{z}=\sigma_{y z}=\sigma_{x z}$ $=0$. However, if the quantitative order of the length is far greater than that of the width, the problem can be treated as in the plane-strain state when the uniform tension or pressure is exerted in the width direction ( $x$ direction). Then, $\varepsilon_{y}=\varepsilon_{y z}$ $=\varepsilon_{x y}=0$. We will discuss the two kinds of plane problems, respectively.

\section{A. Plane-stress state}

If the scales in $y$ direction and $x$ direction of the nanofilm are of the same quantitative order, and a uniform tension is exerted in the $x$ direction, we can perform a simple tension analysis based on the 3D model to derive the values of elastic moduli.

According to the constitutive relation of the nanofilm, Eq. (28), we obtain

$$
\begin{aligned}
& C_{11} \varepsilon_{x}+C_{12} \varepsilon_{y}+C_{13} \varepsilon_{z}=\sigma_{x}, \\
& C_{12} \varepsilon_{x}+C_{11} \varepsilon_{y}+C_{13} \varepsilon_{z}=0, \\
& C_{13} \varepsilon_{x}+C_{13} \varepsilon_{y}+C_{33} \varepsilon_{z}=0 .
\end{aligned}
$$

Solving the simultaneous equations (35b) and (35c), we obtain Poisson's ratios as follows:

$$
\begin{aligned}
& \nu_{x y}=-\frac{\varepsilon_{y}}{\varepsilon_{x}}=\frac{C_{12} C_{33}-C_{13}^{2}}{C_{11} C_{33}-C_{13}^{2}}, \\
& \nu_{x z}=-\frac{\varepsilon_{z}}{\varepsilon_{x}}=\frac{\left(C_{11}-C_{12}\right) C_{13}}{C_{11} C_{33}-C_{13}^{2}} .
\end{aligned}
$$

Substituting Eqs. (36) into Eq. (35a), Young's modulus of the nanofilm in the plane-stress state can be obtained as 
TABLE I. The effect of thickness parameter $\beta$ and relaxation coefficient $k$ the size effect of elastic constants in the $3 \mathrm{D}$ model.

\begin{tabular}{|c|c|c|c|c|c|c|c|c|c|}
\hline & \multicolumn{3}{|c|}{$\beta=\alpha_{2} /\left(\alpha_{1}+2 \alpha_{2}\right)$} & \multicolumn{3}{|c|}{$\beta=0$} & \multicolumn{3}{|c|}{$\beta=1$} \\
\hline & $k>1$ & $k=1$ & $k<1$ & $k>1$ & $k=1$ & $k<1$ & $k>1$ & $k=1$ & $k<1$ \\
\hline$C_{11}$ & $\downarrow$ & - & $\uparrow$ & & $\downarrow$ & & & $\uparrow$ & \\
\hline$C_{12}$ & & $\uparrow$ & & $\downarrow$ & - & $\uparrow$ & & $\uparrow$ & \\
\hline$C_{13}$ & & $\downarrow$ & & & $\downarrow$ & & $\downarrow$ & - & $\uparrow$ \\
\hline$C_{33}$ & & $\downarrow$ & & & $\downarrow$ & & $\uparrow$ & - & $\downarrow$ \\
\hline
\end{tabular}

where the sign "- " denotes the size independence of elastic constant, " $\uparrow "$ denotes the increase of elastic constant, " $\downarrow$ " denotes the decrease of elastic constant, and "/" denotes the uncertain values.

$$
E=-\frac{\sigma_{x}}{\varepsilon_{x}}=\frac{\left(C_{11}-C_{12}\right)\left(C_{11} C_{33}+C_{12} C_{33}-2 C_{13}^{2}\right)}{C_{11} C_{33}-C_{13}^{2}} .
$$

It is evident that both Young's modulus $E$ and Poisson's ratios $\nu_{x y}$ and $\nu_{x z}$ depend on the number of atomic layers and relaxation coefficient $k$. In addition, Young's modulus also depends on the thickness parameter $\beta$. The variations of Young's modulus and Poisson's ratios with the number of atomic layers are shown in Figs. 9 and 10. It can be seen that the results are the same as those of Sun and Zhang ${ }^{21}$ when the relaxation coefficient $k=1$ and the parameter $\beta=0$.

\section{B. Plane-strain state}

To a long striplike nanofilm, when the length is far longer than the width and the uniform tension is exerted in the width direction ( $x$ direction), the nanofilm can be treated as in the plane-strain state. We have $\varepsilon_{y}=\varepsilon_{y z}=\varepsilon_{x y}=0$. The interactions of atoms in the $x-y$ surface are neglected. Thus, the problem can be greatly simplified and a 2D model can be used to derive the elastic moduli of the nanofilm. In fact, a similar simplification was also made in Ref. 18.

A 2D model of the nanofilm is shown in Fig. 11, which is similar to the above 3D model but neglecting the interaction of in-plane atoms. The coordinate axis $x$ is in the middle surface and $z$ perpendicular to the surface (Fig. 11). There are $2(N+1)$ layers of atoms along the thickness. The atomic layers between the $-(N-1)$ th and the $(N-1)$ th layers are uniform with a constant lattice distance $a$. The distance of atoms at the topmost and bottommost layers is $k a$. Each atom is assumed to interact with its nearest- and next-nearest

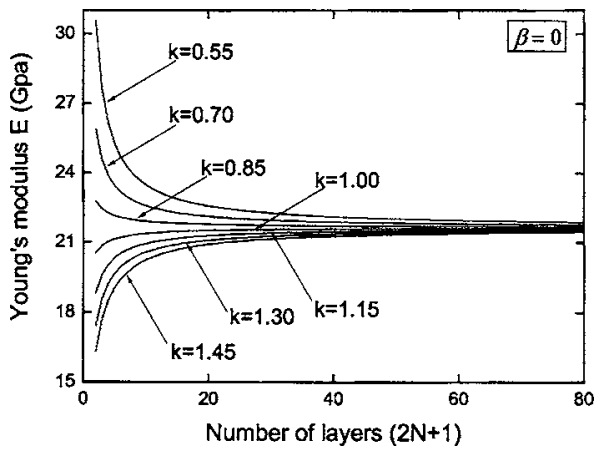

FIG. 9. The variations of Young's modulus $E$ with the number of atomic layers with respect to different values of relaxation coefficient when the thickness parameter $\beta=0$.

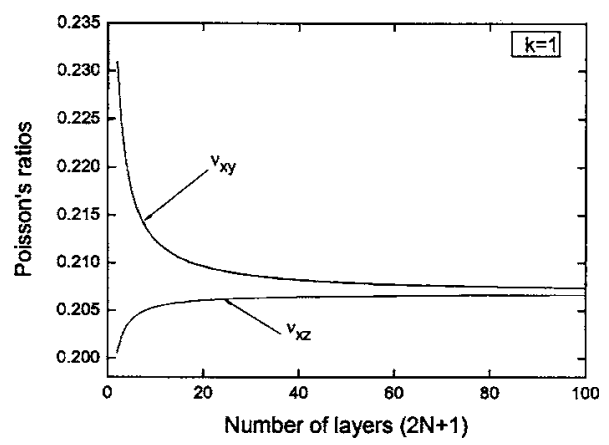

FIG. 10. The variations of Poisson's ratios with the number of atomic layers when the relaxation coefficient $k=1$.

neighbors, but the interactions with in-plane atoms are neglected. The interactions are, respectively, represented by springs with spring constants $\alpha_{1}, \alpha_{2}, \alpha_{3}$, and $\alpha_{4}$ (as shown in Fig. 12). There are three kinds of typical representative elements in the nanostructure (Fig. 12). Type $A$ represents the element in the layers from the $-(N-1)$ th and the $(N-2)$ th layers, type $B$ represents the element at the $(-N)$ th and the $(N-1)$ th layers, and type $C$ is the cell at the topmost layer. The deformation energies of three types of elements are, respectively, expressed as $U_{(i j)}^{A}, U_{(i j)}^{B}$, and $U_{(i N)}^{C}$. Similar to the calculation in Sec. II, the deformation energies of three types of elements are

$$
\begin{aligned}
U_{(i j)}^{A}= & U_{1-2}^{A}+U_{1-3}^{A}+U_{1-4}^{A}+U_{2-3}^{A} \\
= & \frac{1}{2} \alpha_{1} a^{2}\left(\varepsilon_{x}^{2}+\varepsilon_{z}^{2}\right)+\frac{1}{2} \alpha_{2} a^{2}\left[\left(\varepsilon_{x}+\varepsilon_{z}\right)^{2}+\gamma_{x z}^{2}\right] \\
U_{(i j)}^{B}= & U_{1-2}^{B}+U_{1-3}^{B}+U_{1-4}^{B}+U_{2-3}^{B}=\frac{1}{2} a^{2}\left(\alpha_{1} \varepsilon_{x}^{2}+k^{2} \alpha_{3} \varepsilon_{z}^{2}\right) \\
& +\frac{\alpha_{4} a^{2}}{1+k^{2}}\left[\left(\varepsilon_{x}+k^{2} \varepsilon_{z}\right)^{2}+k^{2} \gamma_{x z}^{2}\right]
\end{aligned}
$$

Then the total deformation energy of the nanofilm can be obtained as the sum of all elements

$U_{\text {tot }}=\sum_{i} \sum_{j}^{-(N-1) \leqslant j \leqslant(N-2)} U_{(i j)}^{A}+\sum_{i} \sum_{j}^{2} U_{(i j)}^{B}+\sum_{i} U_{(i N)}^{C}$,

where the contribution energy from the rightmost layer is neglected due to small thickness.

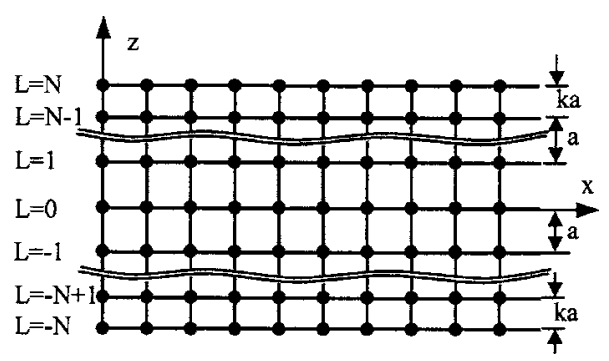

FIG. 11. The schematic of 2D model of the nanofilm in plane-strain state. 


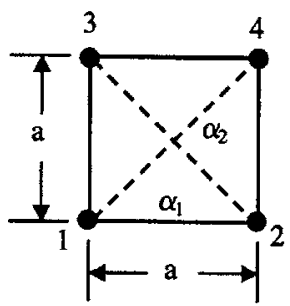

(a). Type A

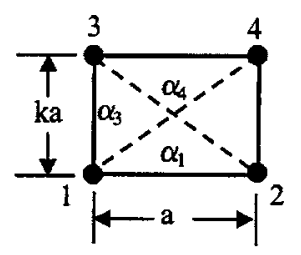

(b). Type B

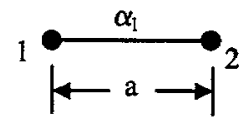

(c). Type C
FIG. 12. The schematics of three kinds of $2 \mathrm{D}$ typical lattice elements: (a) the element in the $j$ th layer $(-N<j<N-1)$, (b) the element in the $(N$ $-1)$ th and the $-N$ th layer, and (c) the element in the most top (i.e., $N$ th) layer.

The average strain energy density can be calculated by Eq. (25). If we choose a crystal lattice as a volume element, i.e., $V_{0}=a \times a \times h$, and the definition of thickness $h$ is the same as that in Sec. II, then the strain energy density function $f$ of this volume is

$$
\begin{aligned}
f= & \frac{1}{[2(N+k)-1-\beta] a}\left[(N-1) \alpha_{1}\left(\varepsilon_{x}^{2}+\varepsilon_{z}^{2}\right)+(N-1)\right. \\
& \left.\times \alpha_{2}\left(\varepsilon_{x}+\varepsilon_{z}\right)^{2}+\frac{3}{2} \alpha_{1} \varepsilon_{x}^{2}+k^{2} \alpha_{3} \varepsilon_{z}^{2}+\frac{2 \alpha_{4}}{1+k^{2}}\left(\varepsilon_{x}+k^{2} \varepsilon_{z}\right)^{2}\right] .
\end{aligned}
$$

When the uniform tension is exerted in the $x$ direction of the nanofilm, we have $\sigma_{z}=\sigma_{x z}=0$. As $\sigma_{z}=\partial f / \partial \varepsilon_{z}=0$, the Poisson's ratio $\nu$ of the nanofilm in the plane-strain state can be written in the following form:

$$
\nu=\frac{2(N-1) \alpha_{2}+\left(4 k^{2} \alpha_{4} / 1+k^{2}\right)}{2(N-1)\left(\alpha_{1}+\alpha_{2}\right)+2 k^{2} \alpha_{3}+\left(4 k^{4} \alpha_{4} / 1+k^{2}\right)} .
$$

Furthermore, substituting Eq. (43) into $\sigma_{x}=\partial f / \partial \varepsilon_{x}$, Young's modulus $E$ of the nanofilm can be obtained as

$$
\begin{aligned}
E= & \frac{1}{[2(N+k)-1-\beta] a}\left\{\left[(2 N+1) \alpha_{1}+2(N-1) \alpha_{2}\right.\right. \\
& \left.+\frac{4 \alpha_{4}}{1+k^{2}}\right] \\
& \left.-\frac{\left[2(N-1) \alpha_{2}+\left(4 k^{2} \alpha_{4} / 1+k^{2}\right)\right]^{2}}{2(N-1)\left(\alpha_{1}+\alpha_{2}\right)+2 k^{2} \alpha_{3}+\left(4 k^{4} \alpha_{4} / 1+k^{2}\right)}\right\} .
\end{aligned}
$$

It is evident that both Young's modulus and Poisson's ratio are dependent on the variable $N$ and the parameters $k$. In addition, Young's modulus also depends on the parameter $\beta$. When the parameter $k$ is equal to 1 , Poisson's ratio $\nu$ is size independent. Similar to the discussion in Sec. II, the characteristic value of the parameter $\beta$, which makes Young's modulus $E$ size independent when $k=1$, can be calculated as

$$
\beta_{1}=\frac{\alpha_{1}}{\alpha_{1}+2 \alpha_{2}} .
$$

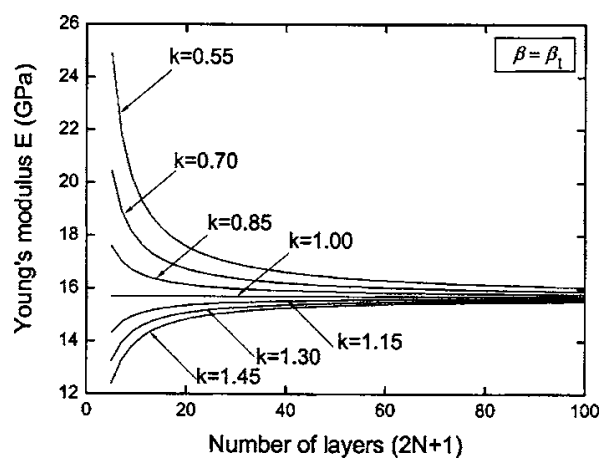

FIG. 13. The variations of Young's modulus $E$ with the number of atomic layers with respect to different values of relaxation coefficient when the thickness parameter $\beta=\beta_{1}$.

When the parameter $\beta$ is equal to $\beta_{1}$, Young's modulus is size independent if the relaxation coefficient $k=1$ and increases with the decrease of atomic layer number if $k<1$ and decreases with the decrease of atomic layer number if $k>1$. The results are shown in Figs. 13 and 14. When the number of atomic layers is less than about 50, Young's modulus and Poisson's ratio vary with the atomic layer number. Especially, they vary sharply as the atomic layer numbers are much less than 20. On the other hand, it is shown from the figures that Young's modulus and Poisson's ratio approach constant values as the number of atomic layers tends to infinity. In fact, when $N \rightarrow \infty$, the Poisson's ratio and Young's modulus can be easily obtained from Eqs. (43) and (44)

$$
\nu=\frac{\alpha_{2}}{\alpha_{1}+\alpha_{2}}, \quad E=\frac{1}{a} \frac{\alpha_{1}^{2}+2 \alpha_{1} \alpha_{2}}{\alpha_{1}+\alpha_{2}},
$$

which only depend on the elastic stiffness $\alpha_{1}$ and $\alpha_{2}$. In addition, since $\alpha_{1}$ and $\alpha_{2}$ denotes the elastic stiffness of the nearest neighbors and the next-nearest neighbors, respectively, it is evident that the value of $\alpha_{1}$ is always greater than that of $\alpha_{2}$. Thus the value of the Poisson's ratio is always less than 0.5 due to the relation $\alpha_{1}+\alpha_{2}>2 \alpha_{2}$.

It can be found from Figs. 13 and 14 that the smaller the relaxation coefficient $k$ is, the greater Young's modulus and Poisson's ratio become when the number of atomic layers is less than about 50. When the number of atomic layers reaches 5 , Young's modulus $E$ increases by $58.6 \%$ of its macroscopic value for $k=0.55$ and decreases by $21.0 \%$ for $k$

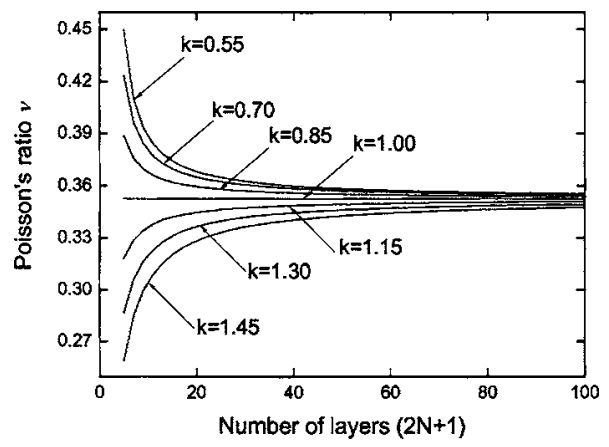

FIG. 14. The variations of Poisson's ratio $\nu$ with the number of atomic layers with respect to different values of relaxation coefficient when the thickness parameter $\beta=\beta_{1}$. 


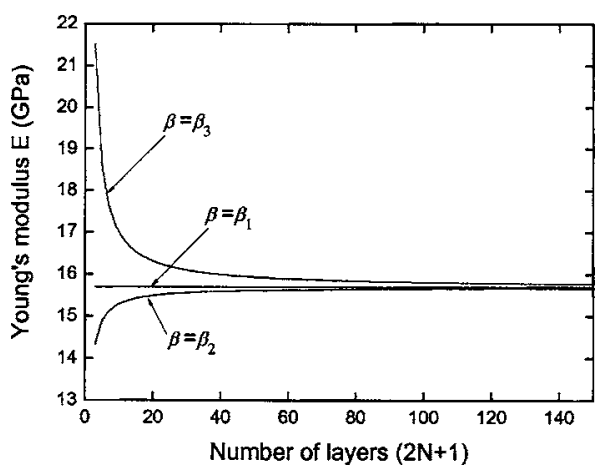

FIG. 15. The variations of Young's modulus $E$ with the number of the atomic layers with respect to different values of $\beta$ when the relaxation coefficient $k=1$

$=1.45$. Similarly, the value of Poisson's ratio $\nu$ increases by $28.6 \%$ of its macroscopic value for $k=0.55$, and decreases by $25.7 \%$ for $k=1.45$.

The definition of the thickness of the nanofilm $h$ does not have an influence on Poisson's ratio, but it bears a close relation with Young's modulus, which is expressed via the variation of the parameter $\beta$. Figure 15 shows the variations of Young's modulus with the decrease of atomic layer number with respect to different values of $\beta$ when the surface relaxation is neglected, i.e., $k=1$. When $\beta$ is equal to $\beta_{1}$, Young's modulus $E$ keeps constant. When $\beta$ reaches its minimum value, i.e., $\beta=\beta_{2}=0$, Young's modulus decreases with the decrease of atomic layer numbers. On the other hand, Young's modulus increases with the decrease of atomic layer numbers when $\beta$ is equal to its maximum value, i.e., $\beta=\beta_{3}$ $=1$, which is similar to the results of Krivtsov and Morozov. ${ }^{18}$ But the influence is negligible as the number of atomic layers is large enough. These results are summarized in Table I, where the meaning of the signs is the same as those in Table II.

\section{CONCLUSION}

A three-dimensional lattice model is presented with the surface relaxation taken into consideration for the sizedependent elastic constants of a nanofilm. A parameter $\beta$ is introduced to define the ambiguity of the thickness of the nanofilm. The interactions between every atom and its nearest- and next-nearest neighbors are considered, and the surface relaxation is considered by introducing a relaxation coefficient $k$ to modify the lattice constant of surface atoms. By calculating the deformation energy of all atomic pairs, the strain energy density is derived. Furthermore, the constitu-

TABLE II. The effect of thickness parameter $\beta$ and relaxation coefficient $k$ the size effect of Young's modulus and Poisson's ratio in the 2D model.

\begin{tabular}{|c|c|c|c|c|c|}
\hline & \multicolumn{3}{|c|}{$\beta=\alpha_{2} /\left(\alpha_{1}+2 \alpha_{2}\right)$} & \multirow{2}{*}{$\begin{array}{l}\beta=0 \\
k=1\end{array}$} & \multirow{2}{*}{$\begin{array}{l}\beta=1 \\
k=1\end{array}$} \\
\hline & $k>1$ & $k=1$ & $k<1$ & & \\
\hline E & $\downarrow$ & - & $\uparrow$ & $\downarrow$ & $\uparrow$ \\
\hline$v$ & $\downarrow$ & - & $\uparrow$ & - & - \\
\hline
\end{tabular}

Where the sign "-_" denotes the size independence of elastic constant, " $\uparrow$ " denotes the increase of elastic constant, and " $\downarrow$ " denotes the decrease of elastic constant. tive relation of the $3 \mathrm{D}$ nanofilm model is obtained. Due to the effect of the surface relaxation, the nanofilm is anisotropic and has four independent elastic constants. These elastic constants are not only size dependent but also dependent on the relaxation coefficient $k$ and the thickness parameter $\beta$. When the value of $k$ is specified as to be equal to 1 , three characteristic values of $\beta$ are derived to make the four elastic constants size independent, respectively. With respect to each characteristic value of $\beta$, certain elastic constant increases or decreases with the decrease of atomic layer number if $k>1$ or $k<1$.

The elastic moduli of the nanofilm in two kinds of plane problems are derived and discussed for the sake of simplification. The elastic moduli of the nanofilm in the plane-stress state are obtained based on the 3D model in the case of simple tension. A simplified 2D model is also presented to study the elastic moduli of the nanofilm in the plane-strain state, and the analytical expressions of Young's moduli and Poisson's ratio are derived. Young's modulus depends on the surface relaxation and the thickness parameter $\beta$, but Poisson's ratio is independent of the parameter $\beta$. When the thickness parameter $\beta$ reaches its characteristic value $\beta_{1}$, the values of Young's modulus and Poisson's ratio decrease with the decrease of the number of atomic layers if the relaxation coefficient $k>1$, while they increase with the decrease of the number of atom layers if the relaxation coefficient $k<1$. In addition, when $k=1$, Young's modulus increases as $\beta>\beta_{1}$ and decreases as $\beta<\beta_{1}$.

\section{ACKNOWLEDGMENTS}

This research was supported by the National Natural Science Foundation of China (NSFC) (Grant Nos. 10225209 and 90305020), key project from the Chinese Academy of Sciences (Grant No. KJCX2-SW-L2), and NSFC-RGC Joint Project (Grant No. 50131160739).

${ }^{1}$ H. G. Craighead, Science 290, 1532 (2000).

${ }^{2}$ J. A. Ruud, T. R. Jervis, and F. Spaepen, J. Appl. Phys. 75, 4969 (1994).

${ }^{3}$ Y. Choi, K. J. van Vliet, J. Li, and S. Suresh, J. Appl. Phys. 94, 6050 (2003).

${ }^{4}$ J. Wang, F. G. Shi, T. G. Nieh, B. Zhao, M. R. Brongo, S. Qu, and T. Rosenmayer, Scr. Mater. 42, 687 (2000).

${ }^{5}$ D. Son, J. Jeong, and D. Kwon, Thin Solid Films 437, 182 (2003).

${ }^{6}$ S. Sundararaian and B. Bhushan, Sens. Actuators, A 101, 338 (2002).

${ }^{7}$ S. Cuenot, C. Frétigny, S. D. Champagne, and B. Nysten, Phys. Rev. Lett. 85, 1690 (2000).

${ }^{8}$ S. Cuenot, S. D. Champagne, and B. Nysten, Phys. Rev. B 69, 165410 (2004).

${ }^{9}$ M. C. Salvadori, I. G. Brown, A. R. Vaz, L. L. Melo, and M. Cattani, Phys. Rev. B 67, 153404 (2003).

${ }^{10}$ M. C. Salvadori, A. R. Vaz, L. L. Melo, and M. Cattani, Surf. Rev. Lett. 10, 571 (2003).

${ }^{11}$ P. O. Renault, E. Le Bourhis, P. Villain, P. Goudeau, K. F. Badawi, and D. Faurie, Appl. Phys. Lett. 83, 473 (2003).

${ }^{12}$ P. Villain, P. Goudeau, P. O. Renault, and K. F. Badwi, Appl. Phys. Lett. 81, 4365 (2002)

${ }^{13}$ X. X. Li, T. Ono, Y. Wang, and M. Esashi, Appl. Phys. Lett. 83, 3081 (2003).

${ }^{14}$ K. E. Petersen and C. R. Guarnieri, J. Appl. Phys. 50, 6761 (1979).

${ }^{15}$ D. C. Hurley, V. K. Tewary, and A. J. Richards, Thin Solid Films 398, 326 (2001).

${ }^{16}$ J. W. Chung, C. S. Lee, D. H. Ko, J. H. Han, K. Y. Eun, and K. R. Lee, Diamond Relat. Mater. 10, 2069 (2001).

${ }^{17}$ C. W. Nan, X. P. Li, K. F. Cai, and J. Z. Tong, J. Mater. Sci. Lett. 17, 1917 
(1998).

${ }^{18}$ A. M. Krivtsov and N. F. Morozov, Phys. Solid State 44, 2260 (2002).

${ }^{19}$ L. H. Liang, J. C. Li, and Q. Jiang, Solid State Commun. 121, 453 (2002)

${ }^{20}$ P. Zhang, Y. Huang, P. H. Geubelle, P. A. Kevin, and K. C. Hwang, Int. J. Solids Struct. 39, 3893 (2002).

${ }^{21}$ C. T. Sun and H. Zhang, J. Appl. Phys. 93, 1212 (2003).

${ }^{22}$ H. Zhang and C. T. Sun, AIAA J. 42, 2002 (2004).

${ }^{23}$ K. Van Workum and J. J. de Pablo, Phys. Rev. E 67, 031601 (2003).

${ }^{24}$ P. Sharma and S. Ganti, J. Mater. Res. 18, 1823 (2003).

${ }^{25}$ F. Q. Yang, J. Appl. Phys. 95, 3516 (2004).

${ }^{26}$ R. E. Miller and V. B. Shenoy, Nanotechnology 11, 139 (2000).
${ }^{27}$ F. H. Streitz, R. C. Cammarata, and K. Sieradzki, Phys. Rev. B 49, 10699 (1994).

${ }^{28}$ F. H. Streitz, R. C. Cammarata, and K. Sieradzki, Phys. Rev. B 49, 10707 (1994).

${ }^{29}$ J. Q. Broughton and C. A. Meli, Phys. Rev. B 56, 611 (1997).

${ }^{30}$ P. Villain, P. Beauchamp, K. F. Badwi, P. Goudeau, and P. O. Renault, Scr. Mater. 50, 1247 (2004).

${ }^{31}$ L. G. Zhou and H. Huang, Appl. Phys. Lett. 84, 1940 (2004).

${ }^{32}$ P. J. Feibelman, Phys. Rev. B 53, 13740 (1996).

${ }^{33}$ C. Q. Sun, B. K. Tay, X. T. Zeng, S. Li, T. P. Chen, J. Zhou, H. L. Bai, and E. Y. Jiang, J. Phys.: Condens. Matter 14, 7781 (2002). 